\title{
Volatile Compounds in the Leaf of Plane Tree (Platanus orientalis) with Solid Phase Microextraction (SPME) Technique
}

\author{
Zehra GÜLER *1, Ahmet DURSUN¹, Dilek ÖZKAN ${ }^{1}$ \\ ${ }^{1}$ Mustafa Kemal University, Faculty of Agriculture, Food Engineering Department, Hatay/TURKEY
}

Received: 05 May 2017 - Revised: 18 June 2017 - Accepted: 08 August 2017

\begin{abstract}
Plane tree belongs to Platanaceae family. There is a widespread belief that Platanus orientalis leaves (POLs) have beneficial effects on joint disorders. Therefore, many people consume POLs as tea. To our knowledge, as there is no study on volatile compounds (VCs) of POLs, we objected to determine the VCs in POLs obtained from Platanus orientalis trees grown in Hatay province, Turkey. The VCs were extracted using solid phase micro-extraction (SPME) and analyzed by gas chromatography-mass spectrometry (GC-MS). A total of 140 VCs were found in POLs. Aldehydes, alcohols, ketones, terpenes and alkenes were determined in POLs as major VCs groups, which accounted for $32.40 \%, 23.51 \%, 18.08 \%, 10.24 \%$ and $4.82 \%$ of total VCs identified, respectively. Trans, trans-2,4-heptadienal (6.62\%), nonanal (6.46\%), benzaldehyde (6.42\%), cis-3-hexen-1-ol $(6.32 \%)$, benzenemethanol $(6.13 \%)$ were the most abundant VCs identified in POLs. Trans-2-hexenal (3.46\%), 3-phenyl-2-butanone (2.87 \%), trans-3,5-dimethyl-1,6-octadiene (2.80\%), 6-methyl-5-hepten-2-one (2.56\%), octan-1-ol (2.43\%), trans-geranyl acetone (2.17\%), trans-4,8-dimethyl-1,3,7-nonatriene (1.98\%), phenyl methyl ketone (1.69\%), 6-methyl-3,5-heptadiene-2-one (1.57\%) were the second most plentiful compounds found in POLs. 11H-dibenzo[b,e][1,4]diazepin-11-one,5,10-dihydro-5-[3-(methylamino)propyl] (1.38\%), benzeneethanol $(1.36 \%)$ and $\beta$-ionone $(1.02 \%)$ were found as the third most abundant VCs. The above-mentioned VCs were accounted for about $57 \%$ of total VCs identified in POLs. The remaining VCs were below $1.00 \%$ that is, found at trace levels. According to the VCs profiles of POL, its beneficial effects on health may be due to aldehydes, alcohols, ketones and terpenes.
\end{abstract}

Keywords: Platanus orientalis leaf, Volatile compounds, SPME

\section{INTRODUCTION}

Plane tree belonging to Platanaceae family is known for its grandeur and longevity. Platanus orientalis is one of the most common plane varieties in the world and it is the most widespread plane tree growing in Turkey. The common name of this variety is oriental plane. Among plane trees, it was the first variety to be discovered. The oriental plane is widely used as an ornamental, especially in urban areas and also is cultivated sometimes for timber. Plane tree roots have been used as a hemostatic agent and antivenom for snake bite [1]. The pollens of plane tree are an important source of airborne allergens such as asthma, allergic rhinitis and allergic conjunctivitis [2,3] and also systemic reactions [4]. Nevertheless, plane leaves are used

*Corresponding Author E-mail: zguler@mku.edu.tr

ISSN: 2148-6905 online /C) 2017

DOI: $10.21448 / \mathrm{ijsm} .369776$ 
in traditional medicine, especially traditional Persian folk medicine, to treat several disorders such as dermatological, gastrointestinal, rheumatic and inflammatory [5-6]. Recently, the tea of plane tree leaf has widely been used for treating joint pains in the folk medicine in Turkey. It has indicated that the extract of leaves of plane tree resulted in a favorable effect on the removing the symptoms of food allergy in dogs [6]. According to Persian scientists and hakims, people suffer from joint pains such as rheumatism and arthritis or from teeth pain can cope with these pains with methods that are completely natural and herbal [1]. However, it is not known that what compounds are responsible for removing the symptoms of food allergy.

Previous chemical investigations of American sycamore (Platanus occidentalis L.) have shown the presence of triterpenoids or flavonoids which are probably responsible for its antiinflammatory and antinociceptive activity [8]. It is known very-well that treating effect of plants is related to their chemical or biochemical composition. Some volatile compounds such as terpenes, aldehydes, alcohols, ketones occur in plants as a result of the mostly biochemical metabolic pathways. For example, volatile terpene compounds (citronellal, linalool, $\beta$ cubebene, $\beta$-pinene, myrcene, limonene, $\gamma$-terpinene, $\rho$-cymene, terpinolene, copaene, caryophyllene, citronellyl acetate, citronellol, geranyl acetate and $\delta$-cadinene) isolated from lime leaf were found to be effective in inhibiting tumors in the digestive tract [9] and had good antioxidant properties [10]. On the other hand, mono- and sesquiterpenes have been used in studies of geographic variation within the species in wild populations [11]. In general, fragrant and volatile compounds from plants have been used in food industry as additives, medicines and aromatherapy [12].

Based on the common uses of Platanus orientalis leaves in traditional folk medicine, in the present study we were objected to determine the volatile compounds profile of Platanus orientalis leaf.

\section{MATERIAL and METHODS}

The leaves were collected from plane trees about twenty years in Harbiye region of Hatay province. The leaves dried under normal atmospheric condition in summer. VCs analyze of leaves was performed according to the procedures described by Guler [13] with minor modification. Dried samples were cut into in small pieces in cold mortar and taken into headspace vials (Agilent, Palo Alto, CA, USA). Three $\mathrm{mL} \mathrm{NaCl}$ solution ( $3 \%$ concentration) was added in samples. The adsorption of VCs was carried out using Solid Phase Microextraction Technique (SPME) by a divinylbenzene/carboxen/polydimetylsilioxan fiber (Supelco, Bellefonte, PA, USA). The extraction of VCs was done in water bath at $60{ }^{\circ} \mathrm{C}$. The samples were hold in water bath for $45 \mathrm{~min}$ than fiber was inserted to headspace vial and kept at $30 \mathrm{~min}$. For desorption of VCs, fiber hold in injection port at $250{ }^{\circ} \mathrm{C}$ for $5 \mathrm{~min}$. The VCs were analyzed by gas chromatography-mass spectrometry (GC-MS) using HP-Innowax capillary column (60 m x $0.25 \mathrm{~mm}$ id $\times 0.25 \mu \mathrm{m}$ film thickness). The oven temperature program was initially held at $50{ }^{\circ} \mathrm{C}$ for $5 \mathrm{~min}$ and then programmed from $50{ }^{\circ} \mathrm{C}$ by a ramp of $5^{\circ} \mathrm{C} \mathrm{min}-1$ up to $230^{\circ} \mathrm{C}$, which was held for $5 \mathrm{~min}$. Peak identification was carried out by comparing the mass spectra with the NIST (National Institute of Standards and Technology, Gaithersburg, MD, USA) library, version 0.2 L. Peak areas (arbitrary units) were calculated from the total ion current. The results from the VCs analyses were expressed as the percentage composition of each compound.

\section{RESULTS and DISCUSSIONS}

A total of 140 volatile compounds including aldehydes (25), ketones (25), alcohols (21), terpenes (21), phenyls and phenols (15), alkenes (9), alkanes (8), esters (6), furans (5), others (5; alkyne, acid, compound with sulfur, lactone and unknown) were identified in the leaf of 
Platanus orientalis tree. As shown in Table 1. and Figure 1., aldehydes were the most abundant compounds accounting for $32.4 \%$ of total volatile compounds identified in Platanus orientalis leaf, followed by alcohols (23.5\%), ketones (18\%), terpenes $(10 \%)$, alkenes $(4.8 \%)$, alkanes $(2.8 \%)$, esters $(2.7 \%)$, phenyls and phenols $(2.0 \%)$ and furans $(1.8 \%)$. A total of 17 volatile compounds (Figure 2) including trans,trans-2,4-heptadienal (6.6\%), nonanal (6.5\%), benzaldehyde $(6.4 \%)$, cis-3-hexen-1-ol $(6.3 \%)$ and benzenmethanol $(6.2 \%)$, trans-2-hexenal (3.5\%), 3-phenyl-2-butanone (2.9\%), trans-3,5-dimethyl-1,6-octadiene (2.8\%), 6-methyl-5hepten-2-one (2.7\%), octan-1-ol (2.4\%), trans-geranyl acetone (2.17\%), trans-4,8-dimethyl1,3,7-nonatriene $(2.0 \%)$, phenyl methyl ketone (1.7\%), 6-methyl-3,5-heptadiene-2-one (1.6 $\%), 11 \mathrm{H}$-dibenzo[b,e][1,4]diazepin-11-one-5,10-dihydro-5-[3-(methylamino) propyl] (1.4\%) benzenethanol (phenylethyl alcohol) $(1.4 \%)$ and $\beta$-ionone $(1.0 \%)$ were constituted the majority of volatile compounds, which accounted for $57 \%$ of total volatiles identified in head space of the leaf. These VCs are the mainly products derived from unsaturated fatty acids, carotenoids, phenylpropanoid/benzenoid and terpenes.

The major volatiles, trans, trans-2,4-heptadienal has a fatty and green odor. It retards or prevents the development of 'off-flavors' in autoxidizing fats and oils [14]. Nonanal has a fatty and citrus-like flavor note. It is one of the main constituents in the oils of citrus fruits and also reported in over 200 food and beverages. Cis-3-hexen-1-ol and trans-2-hexenal with an intense grassy-green odor note are known as leaf alcohol and aldehyde, respectively. They act as an attractant to many predatory insects. It was observed that cis-3-hexen-1-ol had a less neurotoxicity effect in rat, compared with nonanal [12]. It is safer than nonanal. 
Table 1. The percentage values of volatile compounds identified in Platanus orientalis leaf.

\begin{tabular}{|c|c|c|c|}
\hline Volatile Compounds (140) & RT & RI & $\mathbf{M E A N} \pm \mathbf{S D}$ \\
\hline \multicolumn{4}{|l|}{ Aldehydes (25) } \\
\hline Hexanal & 10.55 & 842 & $0.12 \pm 0.02$ \\
\hline 2-Pentenal & 12.37 & 1202 & $0.01 \pm 0.01$ \\
\hline trans-Citral & 13.71 & 1262 & $0.82 \pm 0.08$ \\
\hline Heptenal & 14.39 & 1291 & $0.12 \pm 0.03$ \\
\hline trans-2-Hexenal & 15.65 & 1353 & $3.46 \pm 0.40$ \\
\hline Octanal & 18.00 & 1484 & $0.75 \pm 0.11$ \\
\hline trans-2-Heptenal & 19.15 & 1561 & $0.67 \pm 0.32$ \\
\hline Nonanal & 21.19 & 1715 & $6.46 \pm 0.85$ \\
\hline trans,trans-2,4-Hexadienal & 21.64 & 1754 & $0.97 \pm 0.14$ \\
\hline 2-Octenal & 22.30 & 1811 & $0.81 \pm 0.15$ \\
\hline trans,trans-2,4-Heptadienal & 23.33 & 1905 & $6.62 \pm 0.48$ \\
\hline Benzaldehyde & 25.09 & 2072 & $6.42 \pm 0.78$ \\
\hline trans, cis-2,6-Nonadienal & 26.51 & $>2100$ & $0.43 \pm 0.02$ \\
\hline trans-2-Decenal & 27.88 & $>2100$ & $0.59 \pm 0.15$ \\
\hline Safranal & 28.12 & $>2100$ & $0.62 \pm 0.08$ \\
\hline cis-Citral & 28.84 & $>2100$ & $0.14 \pm 0.03$ \\
\hline 2-Hydroxy-benzaldehyde & 29.04 & $>2100$ & $0.18 \pm 0.05$ \\
\hline trans,trans-2,4-Nonadienal & 29.35 & $>2100$ & $0.97 \pm 0.14$ \\
\hline 4-Ethyl-benzaldehyde & 29.67 & $>2100$ & $0.19 \pm 0.07$ \\
\hline 2-Undecenal & 30.42 & $>2100$ & $0.59 \pm 0.14$ \\
\hline trans,cis-2,4-Decadienal & 30.76 & $>2100$ & $0.34 \pm 0.03$ \\
\hline Tridecanal & 31.74 & $>2100$ & $0.34 \pm 0.04$ \\
\hline Myrtenal & 32.71 & $>2100$ & $0.14 \pm 0.03$ \\
\hline Tetradecanal & 36.17 & $>2100$ & $0.54 \pm 0.15$ \\
\hline 4-Methoxy-benzenaldehyde & 36.62 & $>2100$ & $0.06 \pm 0.01$ \\
\hline 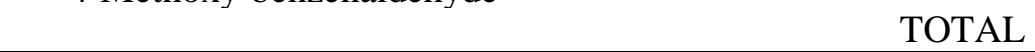 & & & $32.40 \pm 2.73$ \\
\hline \multicolumn{4}{|l|}{ Ketones (25) } \\
\hline 3-Octanone & 16.81 & 1413 & $0.29 \pm 0.07$ \\
\hline 2-Octanone & 17.76 & 1470 & $0.13 \pm 0.03$ \\
\hline 6-Methyl-5-hepten-2-one & 19.51 & 1586 & $2.56 \pm 0.10$ \\
\hline Camphenilone & 19.97 & 1619 & $0.30 \pm 0.09$ \\
\hline Octamethyl-cyclotetrasiloxane & 23.05 & 1879 & $0.12 \pm 0.04$ \\
\hline $\begin{array}{l}\text { 11H-Dibenzo[b,e][1,4]diazepin-11-one, 5,10-Dihydro-5-[3- } \\
\text { (methylamino)propyl] }\end{array}$ & 23.42 & 1913 & $1.38 \pm 0.24$ \\
\hline 4-(2,6,6-trimethyl-2-cyclohexen-1-yl)-,( R)-2-Butanone & 23.49 & 1919 & $0.21 \pm 0.03$ \\
\hline 1,3-Dihydroxy-6-methoxy-1,2,3,4,-tetrahydroquinolin-2-one & 23.94 & 1960 & $0.19 \pm 0.02$ \\
\hline 3,5-Octadien-2-one & 24.77 & 2042 & $0.24 \pm 0.07$ \\
\hline 4,8-Dimethyl-nona-3,8-dien-2-one & 25.66 & $>2100$ & $0.15 \pm 0.04$ \\
\hline 3,5-Octadiene-2-one & 26.13 & $>2100$ & $0.14 \pm 0.02$ \\
\hline 6-Methyl-3,5-heptadiene-2-one & 26.70 & $>2100$ & $1.57 \pm 0.25$ \\
\hline Phenyl methyl ketone & 28.31 & $>2100$ & $1.69 \pm 0.50$ \\
\hline Glycocyanidine & 29.27 & $>2100$ & $0.17 \pm 0.03$ \\
\hline 2-Hydroxy acetophenone & 31.86 & $>2100$ & $0.10 \pm 0.01$ \\
\hline$\beta$-Damascenone & 32.09 & $>2100$ & $0.10 \pm 0.04$ \\
\hline trans-Geranyl acetone & 32.63 & $>2100$ & $2.17 \pm 0.11$ \\
\hline 6-Methyl-5-hepten-2-one & 32.80 & $>2100$ & $0.34 \pm 0.17$ \\
\hline 3-Phenyl-2-butanone & 33.01 & $>2100$ & $2.87 \pm 0.07$ \\
\hline 6,10-Dimethyl-9-undecen-2-one & 34.42 & $>2100$ & $0.22 \pm 0.02$ \\
\hline$\beta$-Ionone & 34.62 & $>2100$ & $1.02 \pm 0.29$ \\
\hline 2,3 -Epoxy- $\beta$-1onone & 35.78 & $>2100$ & $0.67 \pm 0.09$ \\
\hline 6,10,14-Trimethyl-2-pentadecanone & 38.00 & $>2100$ & $0.70 \pm 0.10$ \\
\hline 3-Ethyl-4-methyl-1H-pyrrole-2,5-dione & 41.01 & $>2100$ & $0.24 \pm 0.04$ \\
\hline $5,6,7,7 \mathrm{a}$-Tetrahydro-4,4,7a-trimethyl-2(4H)-benzofuranone & 43.03 & $>2100$ & $0.50 \pm 0.10$ \\
\hline TOTAL & & & $18.08 \pm 0.20$ \\
\hline
\end{tabular}


Table 1. (Continued).

\begin{tabular}{|c|c|c|c|}
\hline \multicolumn{4}{|l|}{ Alcohols (21) } \\
\hline Hexen-1-ol & 19.79 & 1605 & $0.62 \pm 0.12$ \\
\hline cis-3-Hexen-1-ol & 20.81 & 1684 & $6.32 \pm 0.77$ \\
\hline 1-Octen-3-ol & 22.60 & 1838 & $0.95 \pm 0.07$ \\
\hline 2,6-Dimethyl-7-octen-2-ol & 23.09 & 1883 & $0.13 \pm 0.03$ \\
\hline cis-1,5-Octadien-3-ol & 23.62 & 1931 & $0.56 \pm 0.09$ \\
\hline Octan-1-ol & 25.46 & $>2100$ & $2.43 \pm 0.17$ \\
\hline trans-2-Octen-1-ol & 26.97 & $>2100$ & $0.68 \pm 0.08$ \\
\hline Nonan-1-ol & 27.99 & $>2100$ & $0.56 \pm 0.19$ \\
\hline cis-Geraniol & 31.34 & $>2100$ & $0.59 \pm 0.28$ \\
\hline$\alpha$-Methyl-benzene-methanol & 31.81 & $>2100$ & $0.15 \pm 0.02$ \\
\hline Cuminol & 32.54 & $>2100$ & $0.22 \pm 0.02$ \\
\hline cis-Carveol & 32.88 & $>2100$ & $0.34 \pm 0.17$ \\
\hline Benzene-methanol & 33.25 & $>2100$ & $6.13 \pm 1.46$ \\
\hline Benzene-ethanol & 34.02 & $>2100$ & $1.36 \pm 0.31$ \\
\hline cis-Farnesol & 34.22 & $>2100$ & $0.47 \pm 0.07$ \\
\hline 4-Phenyl-2-butanol & 35.61 & $>2100$ & $0.12 \pm 0.03$ \\
\hline Nerolidol & 36.28 & $>2100$ & $0.98 \pm 0.04$ \\
\hline 2-Methoxy-benzene-ethanol & 36.45 & $>2100$ & $0.14 \pm 0.03$ \\
\hline Octadecan-1-ol & 38.79 & $>2100$ & $0.16 \pm 0.07$ \\
\hline$\left(1 \mathrm{~S}^{*}, 6 \mathrm{~S}^{*}, 7 \mathrm{~S}^{*}\right)$-Tricyclo[5.3.2.0(1,6)]dodecan-7-ol & 38.96 & $>2100$ & $0.55 \pm 0.07$ \\
\hline D11-Dodecene-1-ol & 39.31 & $>2100$ & $0.07 \pm 0.01$ \\
\hline \multicolumn{3}{|c|}{ TOTAL } & $23.51 \pm 1.19$ \\
\hline \multicolumn{4}{|l|}{ Terpenes (21) } \\
\hline \multicolumn{4}{|l|}{ Monoterpenes (10) } \\
\hline dL-Limonene & 14.77 & 1309 & $0.76 \pm 0.07$ \\
\hline$\gamma$-Terpinene & 16.55 & 1398 & $0.81 \pm 0.10$ \\
\hline Styrene & 16.98 & 1423 & $0.65 \pm 0.10$ \\
\hline trans-4,8-Dimethyl-1,3,7-nonatriene & 18.42 & 1510 & $1.98 \pm 0.05$ \\
\hline Theaspirane A & 24.32 & 1995 & $0.30 \pm 0.04$ \\
\hline 9-Hydroxy theaspiran A & 25.32 & 2094 & $0.13 \pm 0.04$ \\
\hline cis- $\alpha$-Bisabolene & 28.91 & $>2100$ & $0.11 \pm 0.01$ \\
\hline Camphene & 29.09 & $>2100$ & $0.34 \pm 0.10$ \\
\hline p-Cymene & 33.39 & $>2100$ & $0.48 \pm 0.15$ \\
\hline \multirow{2}{*}{ Allo ocimene } & \multirow{2}{*}{\multicolumn{2}{|c|}{ TOTAL }} & $0.14 \pm 0.02$ \\
\hline & & & $5.70 \pm 0.24$ \\
\hline \multicolumn{4}{|l|}{ Sesquiterpenes (11) } \\
\hline$\alpha$-Ylangene & 22.98 & 1873 & $0.28 \pm 0.10$ \\
\hline trans-Caryophyllene & 26.88 & $>2100$ & $0.40 \pm 0.11$ \\
\hline$\beta$-Cyclocitral & 27.50 & $>2100$ & $0.85 \pm 0.05$ \\
\hline$\alpha$-Humulene & 28.65 & $>2100$ & $0.94 \pm 0.10$ \\
\hline Zingiberene & 29.57 & $>2100$ & $0.21 \pm 0.08$ \\
\hline$\beta$-Bisabolene & 29.75 & $>2100$ & $0.39 \pm 0.18$ \\
\hline trans,trans- $\alpha$-Farnesene & 30.13 & $>2100$ & $0.24 \pm 0.03$ \\
\hline$\Delta$-Cadinene & 30.54 & $>2100$ & $0.79 \pm 0.16$ \\
\hline trans- $\beta$-Farnesene & 31.01 & $>2100$ & $0.08 \pm 0.04$ \\
\hline $1 \mathrm{~S}$-cis-Calamenene & 32.32 & $>2100$ & $0.26 \pm 0.10$ \\
\hline \multirow[t]{2}{*}{ cis- $\alpha$-Bisabolene epoxide } & 37.50 & $>2100$ & $0.08 \pm 0.02$ \\
\hline & \multicolumn{2}{|l|}{ TOTAL } & $4.53 \pm 0.52$ \\
\hline \multicolumn{4}{|l|}{ Phenyls and Phenols (15) } \\
\hline 1,2,3,4-Tetramethyl-benzene & 17.34 & 1445 & $0.25 \pm 0.10$ \\
\hline 2-Methyl-1,4-benzene-diol & 25.98 & $>2100$ & $0.13 \pm 0.04$ \\
\hline trans-Anethole & 32.23 & $>2100$ & $0.26 \pm 0.12$ \\
\hline 2-Methyl-naphthalene & 33.83 & $>2100$ & $0.18 \pm 0.03$ \\
\hline Phenol & 35.90 & $>2100$ & $0.15 \pm 0.05$ \\
\hline 5-Ethyl-m-xylene & 36.08 & $>2100$ & $0.05 \pm 0.00$ \\
\hline 2-Propenyl-benzene & 36.71 & $>2100$ & $0.17 \pm 0.02$ \\
\hline 2,5-Dimethyl-phenol & 37.10 & $>2100$ & $0.04 \pm 0.01$ \\
\hline
\end{tabular}


Table 1. (Continued).

\begin{tabular}{|c|c|c|c|}
\hline 1-Methyl-5,8-dimethoxy-1,2,3,4-tetrahydro-1,4-1mino- & 37.20 & $>2100$ & $0.07 \pm 0.01$ \\
\hline 2,6-Dimethyl-phenol & 37.31 & $>2100$ & $0.23 \pm 0.03$ \\
\hline m-Cresol & 37.55 & $>2100$ & $0.12 \pm 0.04$ \\
\hline trans-Stilbene & 38.64 & $>2100$ & $0.14 \pm 0.01$ \\
\hline Tymol & 39.80 & $>2100$ & $0.06 \pm 0.01$ \\
\hline Biphenylene & 40.06 & $>2100$ & $0.10 \pm 0.01$ \\
\hline 3-Ethyl-1-naphthol & 40.46 & $>2100$ & $0.09 \pm 0.04$ \\
\hline \multicolumn{4}{|c|}{ TOTAL } \\
\hline \multicolumn{4}{|l|}{ Alkenes (9) } \\
\hline 4-Methyl-2,7-octadiene & 19.39 & 1577 & $0.31 \pm 0.07$ \\
\hline 7-Methyl-3,4-octadiene & 20.17 & 1635 & $0.31 \pm 0.13$ \\
\hline trans-3,5-Dimethyl-1,6-octadiene & 21.46 & 1738 & $2.80 \pm 0.41$ \\
\hline cis-3-Ethyl-2-methyl-1,3-hexadiene & 21.93 & 1779 & $0.12 \pm 0.02$ \\
\hline 2,5-Dimethyl-2,4-hexadiene & 24.54 & 2020 & $0.08 \pm 0.00$ \\
\hline 4,8-Dimethyl-1,7-nonadiene & 25.88 & $>2100$ & $0.13 \pm 0.02$ \\
\hline 4-Methyl-2,6-octadien & 27.06 & $>2100$ & $0.70 \pm 0.26$ \\
\hline $1,2,4,4$-Tetramethyl-cyclopentene & 27.62 & $>2100$ & $0.26 \pm 0.06$ \\
\hline trans- 6-(2-Butenyl)-1,5,5-trimethyl-cyclohexene & 33.54 & $>2100$ & $0.10 \pm 0.02$ \\
\hline 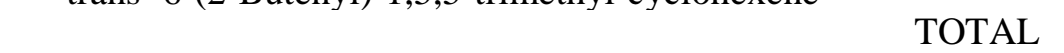 & & & $4.82 \pm 0.51$ \\
\hline \multicolumn{4}{|l|}{ Alkanes (8) } \\
\hline Tetradecane & 21.06 & 1704 & $0.41 \pm 0.05$ \\
\hline 9-Methyl-nonadecane & 22.16 & 1798 & $0.57 \pm 0.16$ \\
\hline Octadecane & 22.75 & 1852 & $0.25 \pm 0.07$ \\
\hline Pentadecane & 23.82 & 1949 & $0.17 \pm 0.07$ \\
\hline Hexadecane & 26.38 & $>2100$ & $0.21 \pm 0.01$ \\
\hline Heptadecane & 28.79 & $>2100$ & $0.71 \pm 0.04$ \\
\hline cis-1,2-Divinylcyclohexane & 30.23 & $>2100$ & $0.32 \pm 0.09$ \\
\hline Cyclododecane & 34.76 & $>2100$ & $0.15 \pm 0.04$ \\
\hline TOTAL & & & $2.78 \pm 0.37$ \\
\hline \multicolumn{4}{|l|}{ Esters (6) } \\
\hline Benzoic acid, 2-[(trimethylsilyl)oxy]-, trimethylsilyl ester & 13.44 & 1250 & $0.42 \pm 0.07$ \\
\hline Tiglic acid, cis-3-hexenyl ester & 28.38 & $>2100$ & $0.28 \pm 0.04$ \\
\hline Acetic acid, phenyl methyl ester & 30.00 & $>2100$ & $0.75 \pm 0.16$ \\
\hline Benzoic acid, 2-hydroxy-, methyl ester & 31.27 & $>2100$ & $0.74 \pm 0.41$ \\
\hline Benzoic acid, cis-3-hexen-1-ol ester & 38.35 & $>2100$ & $0.42 \pm 0.08$ \\
\hline Phthalic acid, ethyl ester & 42.95 & $>2100$ & $0.12 \pm 0.03$ \\
\hline TOTAL & & & $2.72 \pm 0.54$ \\
\hline \multicolumn{4}{|l|}{ Furans (5) } \\
\hline 2-Ethyl-furan & 5.95 & 914 & $0.01 \pm 0.00$ \\
\hline 2-Pentyl-furan & 15.94 & 1367 & $1.00 \pm 0.05$ \\
\hline 2-(2-Propenyl)-furan & 22.37 & 1817 & $0.07 \pm 0.02$ \\
\hline Dihydro- $\beta$-agarofuran & 29.20 & $>2100$ & $0.28 \pm 0.04$ \\
\hline 2-Methyl-5-(1,1,5-trimethyl-5-hexenyl)-furan & 34.88 & $>2100$ & $0.43 \pm 0.11$ \\
\hline 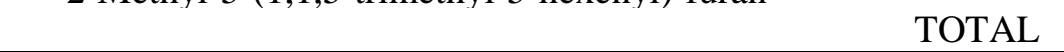 & & & $1.80 \pm 0.11$ \\
\hline \multicolumn{4}{|l|}{ Others (5) } \\
\hline Unknown & 18.92 & 1545 & $0.86 \pm 0.14$ \\
\hline 4-Nonyne & 22.04 & 1788 & $0.25 \pm 0.09$ \\
\hline Ethyl-dimethyl-thiophene & 31.07 & $>2100$ & $0.22 \pm 0.06$ \\
\hline Mint furanone & 34.15 & $>2100$ & $0.07 \pm 0.01$ \\
\hline trans,trans-2,4-Hexadienoic acid & 38.53 & $>2100$ & $0.21 \pm 0.02$ \\
\hline TOTAL & & & $1.61 \pm 0.02$ \\
\hline
\end{tabular}

RI: retention index based on the identified VCs retention time (RT) and calculated from a linear equation between each pair of straight alkanes (C5-C25). 


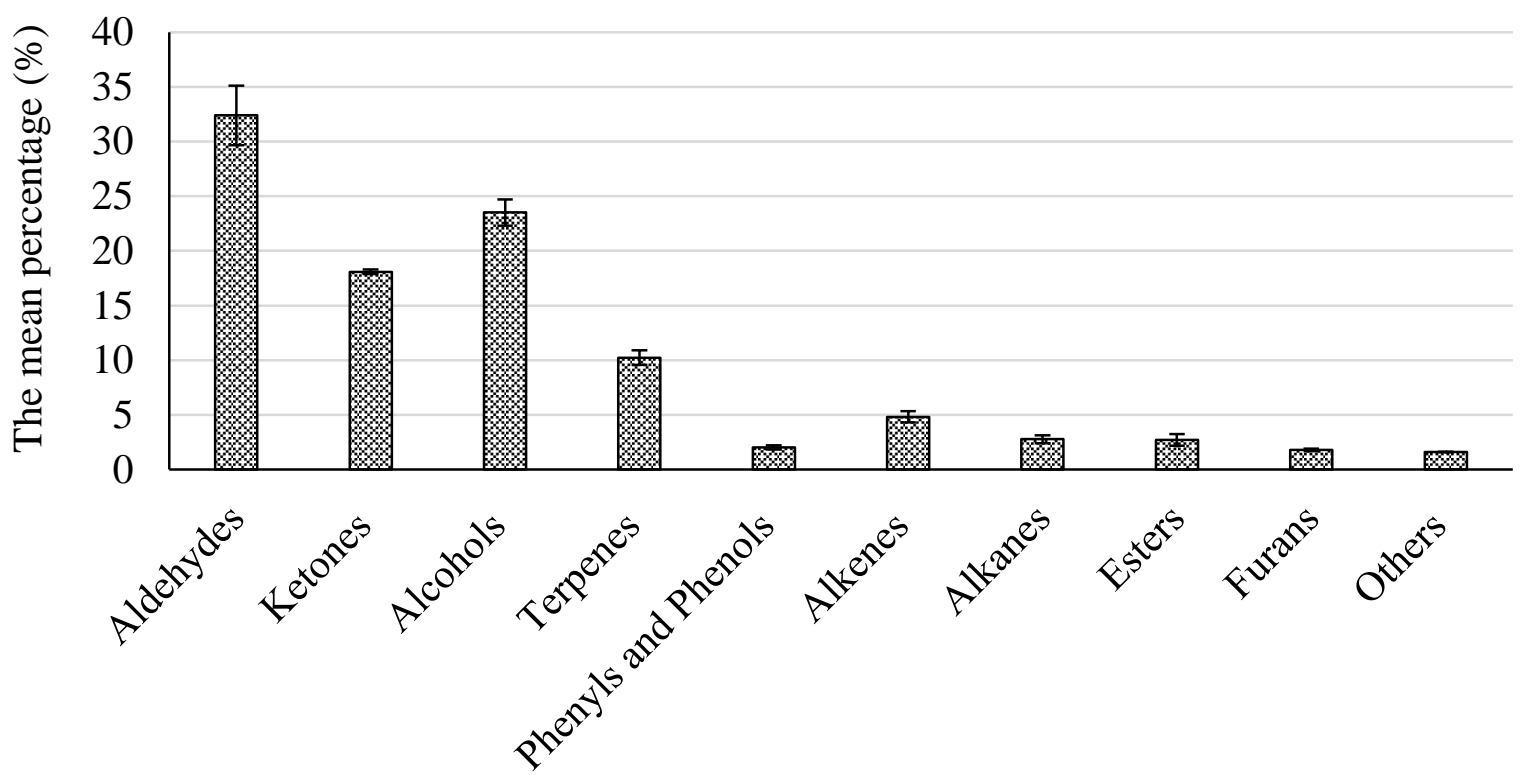

Figure 1. The percentage of volatile compounds identified in Platanus orientalis leaf according to the chemical groups.

Aldehydes, alcohols and also ketone containing six, seven, eight and nine carbons such as cis-3-hexen-1-ol, trans-2-hexenal, trans, trans-2,4-heptadienal, 2-octanone, 1-octanol and nonanal are formed either from linoleic acid or linolenic by oxidative degradation or from $\beta$ scission reactions via unsaturated fatty acids [15]. Glycolipids and phospholipids are rich in linolenic acid and linoleic acids [16]. Octanol or volatiles compounds containing octane give a typical fungal odor. $\beta$-Ionone, benzaldehyde, benzenethanol, cis-3-hexen-1-ol and nonanal were identified in various tea types (white, green e.g.) as main volatile compounds.

Another major VC benzenemethanol is used in perfumes and flavors, dyes for nylon, textiles, and plastics, hair products, plastic packaging and cosmetic industry. But, it is listed as a liver toxicant, neurotoxicant, and immunotoxicant [17]. Benzaldehyde, simplest aromatic aldehyde, is accepted in the European Union as a flavoring agent since it occurs naturally in many foods and is the primary component of bitter almond oil. Benzaldehyde having carcinostatic (anti-cancer) properties does not accumulate in any specific tissues and is metabolized, and then excreted in urine [18]. 


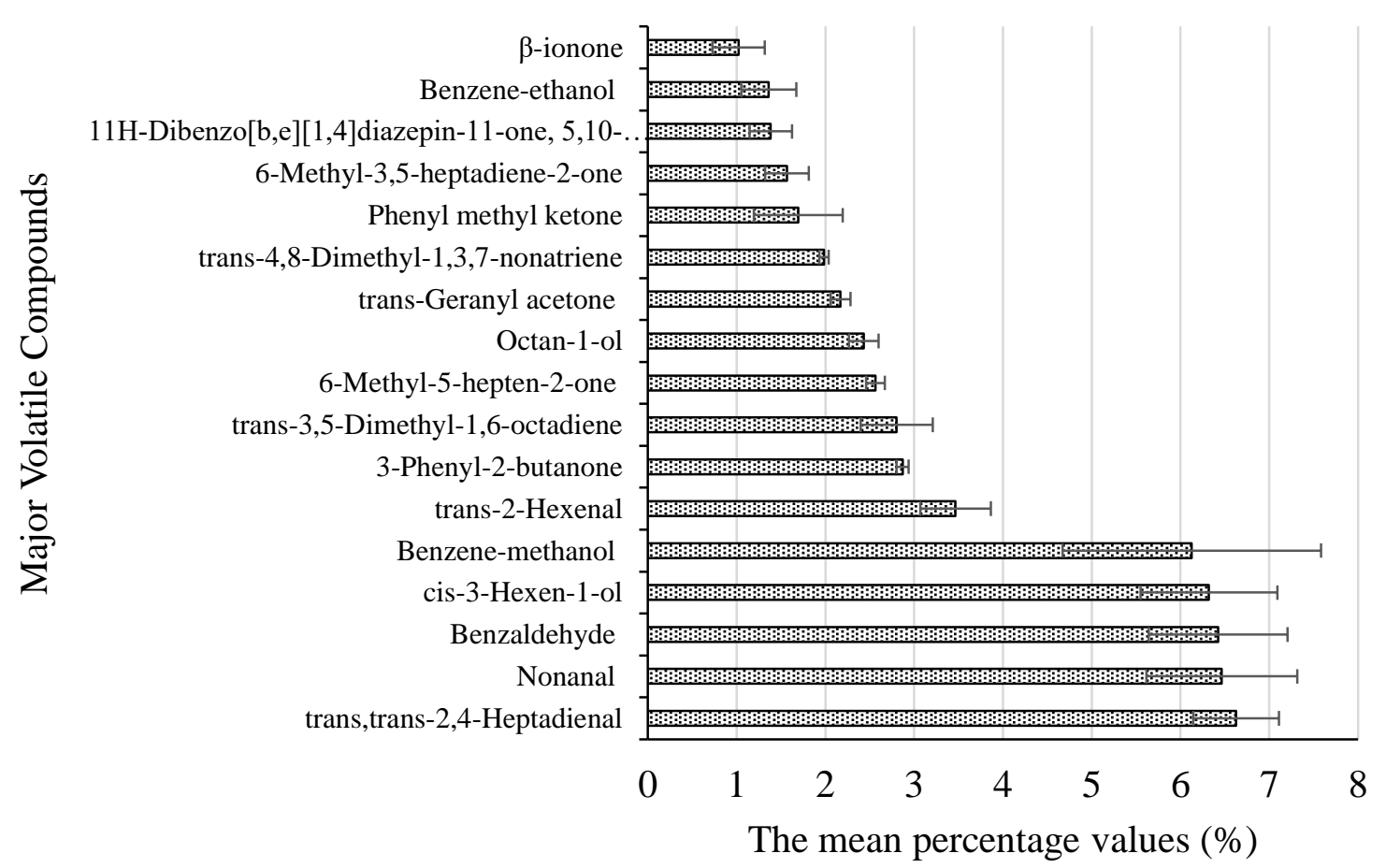

Figure 2. Major volatile compounds identified in Platanus orientalis leaf.

Actually, benzaldehyde, benzenmethanol, 3-phenyl-2-butanone and benzenethanol are phenyl propanoid/benzenoid derivatives [19]. These compounds and also VCs derived from unsaturated fatty acids were more abundant over the other VCs, in terms of their percentages.

Trans-4,8-dimethyl-1,3,7-nonatriene was the most abundant terpene identified in leaf. This compound was found to be in bergamot oil. It was reported that plants synthesize and emit to volatile compounds such as mainly trans-4,8-dimethyl-1,3,7-nonatriene and cis-3-hexen-1$\mathrm{ol}$ in response to insect herbivory [20]. Geranyl acetate is a natural constituent of most essential oils and is used in cosmetic industry as a flavoring agent. $\beta$-Ionone is a product obtained from oxidative degradation of $\beta$-carotene [19]. Ketone 6-methyl-3,5-heptadiene-2-one is a product of lycopene degradation, which is one of main constituents of watermelon flavor [21]. Actually, both $\beta$-carotene and lycopene are terpene. It is well-known that terpenes have therapeutic properties such as antimicrobial and anticancerogenic.

\section{CONCLUSION}

A complex volatile compounds profile including the carotenoid, the unsaturated fatty acid, the terpene, the phenylpropanoid/benzenoid derivatives, the glycoside hydrolysis and the Maillard reaction products (furans) was identified in Platanus orientalis leaves. The diversity of VCs identified indicates that POLs may have a complex chemical and also biochemical composition with the probably majority of phenylpropanoid/benzenoid and unsaturated fatty acids. It is probably that the degradation products derived from phenylpropanoid/benzenoid and unsaturated fatty acids or themselves may be mainly related to the treating effects of Plane tree leaf in folk medicine.

\section{Conflict of Interests}

Authors declare that there is no conflict of interests. 


\section{REFERENCES}

[1]. Nishanbaev, S. Z., Khidyrova, N. K., \& Kuliev, Z. A. (2004). Dimeric proanthocyanidins from Platanus orientalis Bark. Chemistry of Natural Compounds, 40, 93-93.

[2]. Bousquet, J., Hejjaoui, A., Becker, W. M., Cour, P., Chanal, I., Lebel, B., \& Michel, F. B. (1991). Clinical and immunologic reactivity of patients allergic to grass pollens and to multiple pollen species: I. Clinical and immunologic characteristics. Journal of Allergy and Clinical Immunology, 87(3), 737-746.

[3]. Belver, M. T., Caballero, M. T., Contreras, J., Cabañas, R., Sierra, E., Madero, R., \& Serrano, M. L. (2007). Associations among pollen sensitizations from different botanical species in patients living in the northern area of Madrid. Journal of Investigational Allergology \& Clinical Immunology, 17(3), 157-159.

[4]. Hejjaoui, A., Ferrando, R., Dhivert, H., Michel, F. B., \& Bousquet, J. (1992). Systemic reactions occurring during immunotherapy with standardized pollen extracts. Journal of Allergy and Clinical Immunology, 89(5), 925-933.

[5]. Zargari, A. (1990). Medicinal plants (Vol. 4). Tehrari University Publications.

[6]. Torkan, S., Mohajeri, N., \& Khamesipoura, F. (2016). Comparison study of anti food allergy of plane tree leaves extract with the chemical drug therapy in affected dogs. Marmara Pharmaceutical Journal, 20(2), 86-91.

[7]. Haider, S., Nazreen, S., Alam, M. M., Gupta, A., Hamid, H., \& Alam, M. S. (2012). Antiinflammatory and anti-nociceptive activities of Platanus orientalis Linn and its ulcerogenic risk evaluation. Journal of Ethnopharmacology, 143, 236-240.

[8]. Ibrahim, M. A., Mansoor, A. A., Gross, A., Ashfaq, M. K., Jacob, M., Khan, S. I., \& Haman, M. T. (2009). Methicillin-resistance Staphylococcus aureus (MRSA)-active metabolites from Platanus occidentalis (American Sycamore). Journal of Natural Product, 72(12), 2141-2144.

[9]. Murakami, A., Nakamura, Y., Koshimizu, K., \& Ohigashi, H. (1995). Glyceroglycolipids from Citrus hystrix, a traditional herb in Thailand, potently inhibit the tumor promoting activity of 12-o-tetradecanoylphorbol 13-actate in mouse skin. Journal of Agricultural and Food Chemistry, 43(10), 2779-2783.

[10]. Ratseewo, J., Tangkhawanit, E., Meeso, N., Kaewseejan, N., \& Siriamornpun, S. (2016). Changes in antioxidant properties and volatile compounds of kaffir lime leaf as affected by cooking processes. International Food Research Journal, 23, 188-196.

[11]. White, E. E. (1983). Biosynthetic implications of terpene correlations Pinus contorta. Phytochemirrry, 22(6), 1399-1405.

[12]. Yamada, Y., Ohtani, K., Imajo, A., Izu, H., Nakamura, H., \& Shiraishi, K. (2015). Comparison of the neurotoxicities between volatile organic compounds and fragrant organic compounds on human neuroblastoma SK-N-SH cells and primary cultured rat neurons. Toxicology Reports, 2, 729-736.

[13]. Guler, Z. (2014). Profiles of organic acid and volatile compounds in acid-type cheeses containing herbs and spices (surk cheese). International Journal of Food Properties, 17(6), 1379-1392.

[14]. Burdock, G. A. (2005). Fenaroli's Handbook of Flavor Ingredients. 5th edn CRC Press.

[15]. Guler, Z., Karaca, F., \& Yetisir, H. (2013). Volatile compounds in the peel and flesh of cucumber (Cucumis sativus L.) grafted onto bottle gourd (Lagenaria siceraria) rootstocks. The Journal of Horticultural Science \& Biotechnology, 88(2), 123-128.

[16]. Ravichandran, R., \& Parthiban, R. (2000). Lipid occurrence, distribution and degradation to flavour volatiles during tea processing. Food Chemistry, 68, 7-13. 
[17]. Anonymous (2017). URL:

http://www.toxipedia.org/display/toxipedia/Benzenemethanol. Retrieved Feb, 2017.

[18]. Andersen, A. (2006). Final report on the safety assessment of benzaldehyde. International Journal of Toxicology, 25, 11-27.

[19]. Zheng, X. Q., Li, Q. S., Xiang, L. P., \& Liang, Y. R. (2016). Recent advances in volatiles of teas. Molecules, 21(3), 338.

[20]. De Moraes, C. M., Lewis, W. J., Pare, P. W., Alborn, H. T., \& Tumlinson, J. H. (1998). Herbivore-infested plants selectively attract parasitoids. Nature, 393(6685), 570-573.

[21]. Guler, Z., Candır, E., Yetisir, H., Karaca, F., \& Solmaz, I. (2014). Volatile organic compounds in watermelon (Citrullus lanatus) grafted onto 21 local and two commercial bottle gourd (Lagenaria siceraria) rootstocks. Journal of Horticultural Science \& Biotechnology, 89(4), 448-452. 